\title{
2021 Athanasiou Student and Post-Doc Awards
}

\author{
(Published online 21 January 2022)
}

The Kyriacos and Kiley Athanasiou Endowment was established in 2017 within the Biomedical Engineering Society (BMES) to promote biomedical engineering scholarship and honor the contributions of Dr. Kyriacos Athanasiou to the field. One goal of the endowment is to recognize excellence in future leaders of biomedical engineering by awarding the top papers published in the Annals of Biomedical Engineering (ABME) by graduate students and post-doctoral scholars. The fourth Athanasiou Student and PostDoc Award Session was held the week before the 2021 BMES Annual Meeting. Six awardees were selected to present their papers at the virtual session.

The Athanasiou Student and Post-Doc Awards were selected from all papers with graduate student or post-doctoral scholar first authors that were published online in 2020 . There were a total of 90 papers that met the criteria, making the awardees the top $7 \%$ of those papers. The overall impact and quality of papers were considered as well as the number of citations and downloads each paper received. The first author from each paper was contacted to notify them and ask them to present their work during the award session. The papers covered a wide range of topics including tissue engineering and regenerative medicine, injury biomechanics, muskuloskeletal modeling, and medical imaging.

Liu et al. evaluated the accuracy of five instrumented mouthguards commonly used to measure head impact kinematics. ${ }^{8}$ Instrumented mouthguards have shown promise for improved accuracy in measuring head impact biomechanics through tighter coupling with the skull. ${ }^{1,6}$ A pneumatic ram was used to impact a dummy headform fitted with each mouthguard. All mouthguards provided accurate measurements of head impact kinematics and resulting brain injury criteria, with some variation in accuracy by impact location. Mouthguards that provided a long enough sampling window could also be used to estimate brain strain with a convolutional neural network-based brain model.

Modenese and Kohout developed a novel method for automatically generating muscle geometries for subject-specific musculoskeletal models. ${ }^{9}$ Musculoskeletal models can be highly sensitive to subjectspecific parameters. ${ }^{3}$ Muscle geometry is segmented from medical images followed by automated generation of muscle fibers based on a user-defined number of straight-line segments. With validation in a cadaveric dataset, this method shows promise for improving muscle geometries in personalized musculoskeletal models.

D'Costa et al. reviewed recent developments in 3D culture systems. ${ }^{2}$ New biomaterials have enabled production of physiologically relevant organoid or organon-a-chip models. ${ }^{10}$ Induced pluripotent stem cell discovery for use in 3D models has also addressed many ethical considerations for cultured cells. Advances in $3 \mathrm{D}$ culture systems have applications in the areas of fetal development, disease pathology, and drug discovery.

Ding et al. developed an injectable hydrogel to simulate the extracellular matrix microenvironment and stimulate blood vessel regeneration. ${ }^{4}$ Through in vitro and chick embryo chorioallantoic membrane model experiments, the authors showed that the hydrogel loaded with nanoparticles to promote angiogenesis improved blood vessel formation, cell migration, and blood vessel regeneration. In a rat myocardial infarction model, the nanocomposite hydrogel improved recovery of heart function compared with controls. Nanocomposite hydrogels have a variety of applications in regenerative medicine. ${ }^{5}$

Zaffino et al. reviewed different imaging modalities used for intra-operative imaging. ${ }^{11}$ Intra-operative imaging is especially important for the safety and efficacy of minimally-invasive and robot-assisted surgeries. ${ }^{12}$ Imaging modalities all have their own advantages and limitations in terms of operating room compatibility, operator and patient safety, cost, acquisition time, and image characteristics. Advancements in multimodal imaging systems and real-time imaging acquisition will improve surgical techniques and patient outcomes.

Kieffer et al. developed a two-phased approach to evaluating head impact sensors. ${ }^{7}$ In the first phase, accuracy was quantified with the concordance correlation coefficient for laboratory impacts representative of the sensor's intended use. For the second phase, sensors that performed well in laboratory tests were evaluated on the field. The on-field positive predictive value of each sensor was determined through video 
validation of impacts measured by the sensors. For the sensors evaluated, good laboratory performance was not always predictive of good on-field performance. Comprehensive evaluation of head impact sensors can be used to inform users on appropriate use of data collected from those sensors.

The next Athanasiou Student and Post-Doc Award Session will be held at the 2022 BMES Annual Meeting in San Antonio. Papers with graduate student or postdoctoral scholar first authors that were published online in 2021 will be considered for the award. Awardees will be notified by May 1, 2022. Each awardee will receive a $\$ 500$ honorarium and free meeting registration, and will be asked to present their paper at the award session. We look forward to another excellent session showcasing the best graduate student and postdoctoral scholar papers published in ABME.

\section{REFERENCES}

${ }^{1}$ Cecchi, N. J., A. G. Domel, Y. Liu, E. Rice, R. Lu, X. Zhan, Z. Zhou, S. J. Raymond, S. Sami, and H. Singh. Identifying factors associated with head impact kinematics and brain strain in high school American football via instrumented mouthguards. Ann. Biomed. Eng. 49:2814 2826, 2021.

${ }^{2}$ D'Costa, K., M. Kosic, A. Lam, A. Moradipour, Y. Zhao, and M. Radisic. Biomaterials and culture systems for development of organoid and organ-on-a-chip models. Ann. Biomed. Eng. 48:2002-2027, 2020.

${ }^{3}$ Delabastita, T., M. Afschrift, B. Vanwanseele, and F. De Groote. Ultrasound-based optimal parameter estimation improves assessment of calf muscle-tendon interaction during walking. Ann. Biomed. Eng. 48:722-733, 2020.

${ }^{4}$ Ding, Y., A.-S. Zhao, T. Liu, Y.-N. Wang, Y. Gao, J.-A. $\mathrm{Li}$, and $\mathrm{P}$. Yang. An injectable nanocomposite hydrogel for potential application of vascularization and tissue repair. Ann. Biomed. Eng. 48:1511-1523, 2020.

${ }^{5}$ Ding, Y., A.-S. Zhao, T. Liu, Y.-N. Wang, Y. Gao, J.-A. $\mathrm{Li}$, and P. Yang. An injectable nanocomposite hydrogel for potential application of vascularization and tissue repair. Ann. Biomed. Eng. 48(5):1-13, 2020.

${ }^{6}$ Gabler, L. F., N. Z. Dau, G. Park, A. Miles, K. B. Arbogast, and J. R. Crandall. Development of a low-power instrumented mouthpiece for directly measuring head acceleration in American football. Ann. Biomed. Eng. 49:2760-2776, 2021.

${ }^{7}$ Kieffer, E. E., M. T. Begonia, A. M. Tyson, and S. Rowson. A two-phased approach to quantifying head impact sensor accuracy: in-laboratory and on-field assessments. Ann. Biomed. Eng. 48:2613-2625, 2020.

${ }^{8}$ Liu, Y., A. G. Domel, S. A. Yousefsani, J. Kondic, G. Grant, M. Zeineh, and D. B. Camarillo. Validation and comparison of instrumented mouthguards for measuring head kinematics and assessing brain deformation in football impacts. Ann. Biomed. Eng. 48:2580-2598, 2020.

${ }^{9}$ Modenese, L., and J. Kohout. Automated generation of three-dimensional complex muscle geometries for use in personalised musculoskeletal models. Ann. Biomed. Eng. 48:1793-1804, 2020.

${ }^{10}$ Northcutt, L. A., A. Suarez-Arnedo, and M. Rafat. Emerging biomimetic materials for studying tumor and immune cell behavior. Ann. Biomed. Eng. 48:2064-2077, 2020.

${ }^{11}$ Zaffino, P., S. Moccia, E. De Momi, and M. F. Spadea. A review on advances in intra-operative imaging for surgery and therapy: imagining the operating room of the future. Ann. Biomed. Eng. 48:2171-2191, 2020.

${ }^{12}$ Zhao, J.-X., C. Li, H. Ren, M. Hao, L.-C. Zhang, and P.-F. Tang. Evolution and current applications of robot-assisted fracture reduction: a comprehensive review. Ann. Biomed. Eng. 48:203-224, 2020.

\section{BETHANY RowsON (D)}

\section{Virginia Tech}

Electronic mail: browson@vt.edu

Publisher's Note Springer Nature remains neutral with regard to jurisdictional claims in published maps and institutional affiliations. 